\title{
Agôn
}

Revue des arts de la scène

Critiques | Saison 2013-2014

\section{Christian Rizzo : Le bénéfice du doute}

Qu'est-ce qui nous tient encore en mouvement?

\section{Frédérique Villemur}

\section{(2) OpenEdition}

Journals

Édition électronique

URL : http://journals.openedition.org/agon/2713

DOI : 10.4000/agon.2713

ISSN : 1961-8581

Éditeur

Association Agôn

Référence électronique

Frédérique Villemur, «Christian Rizzo : Le bénéfice du doute », Agôn [En ligne], Critiques, mis en ligne le 28 août 2013, consulté le 23 septembre 2020. URL : http://journals.openedition.org/agon/2713 ; DOI : https://doi.org/10.4000/agon.2713

Ce document a été généré automatiquement le 23 septembre 2020

Association Agôn et les auteurs des articles 


\title{
Christian Rizzo : Le bénéfice du doute
}

Qu'est-ce qui nous tient encore en mouvement?

\author{
Frédérique Villemur
}

\section{RÉFÉRENCE}

Le bénéfice du doute : conception, chorégraphie, costumes : Christian Rizzo ; lumière Caty Olive ; musique originale : Robin Rimbaud - Scanner : www.scannerdot.com.

Présenté lors de Montpellier Danse, 2012-2013, au Théâtre La Vignette.

Prochaine tournée : 15 mai 2014, Chassé Theater, Breda, Pays-Bas ; 20 mai 2014, Le

Moulin du Roc, Niort.

1 Il y a un tournant dans la création de Christian Rizzo, que sa dernière production au Festival d'Avignon (D'après une histoire vraie, 2013) est venue confirmer, tournant qui s'est trouvé avec Le bénéfice du doute (2012), après Mon amour (2008) et L'oubli, toucher du bois (2010). Nous revenons sur cette pièce créée à l'Opéra de Lille, où l'association fragile de Christian Rizzo a été en résidence de septembre 2007 à juin 2012, manière de clore un cycle dont on voit qu'il s'ouvre aujourd'hui à de nouveaux possibles.

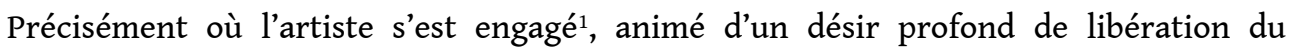
mouvement, voire de transe, et où son obsession plasticienne a fini par céder devant le dénuement du plateau, pour puiser dans la vitalité et la puissance de la danse. «Le jet chorégraphique direct et libéré » remarqué à Avignon ${ }^{2}$, prend sa source dans Le bénéfice $d u$ doute dont le titre souligne, pour Christian Rizzo, que « dès lors que l'on commence à douter, tout peut se mettre en mouvement. Le doute est une promesse de nouveauté $»^{3}$. 


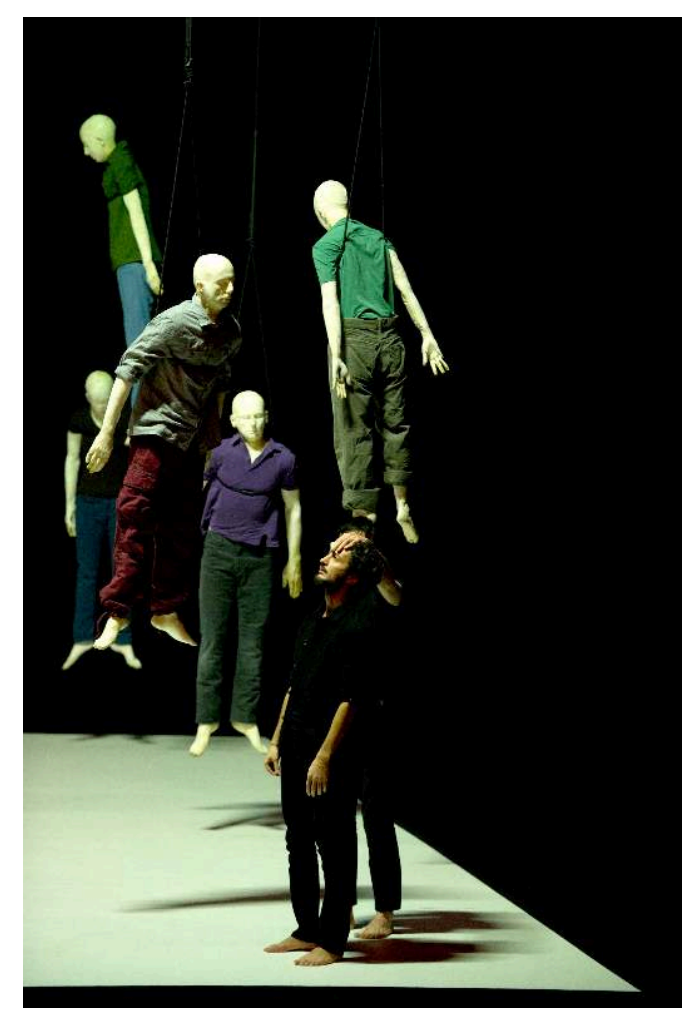

(c) Marc Dommage

2 Le point de départ du Bénéfice du doute, contrairement à tous ses spectacles précédents, n'est pas scénographique (on se souvient de la mise en abîme du théâtre dans le théâtre avec la grande boîte de L'oubli, toucher du bois) : "Je ne souhaite plus faire évoluer [les danseurs] dans un cadre qui leur préexiste. Ma préoccupation est de mettre en scène leurs énergies. Les corps sont désormais livrés à eux-mêmes et doivent se confronter au vide qui les enveloppe; [...] Sans décor tangible, le mouvement des danseurs doit assurer ses propres fondations. L'énergie qu'ils libèrent constitue l'armature de toutes leurs actions $»^{4}$.

Désireux de se défaire de toute image, Rizzo reconnaît à la présence des corps sur scène le magnétisme intact des mouvements qu'ils engendrent. Ce que montre l'introduction de la pièce dans cet inventaire des apparences : les danseurs arrivent sur scène portant des mannequins vêtus de noir avec lesquels ils échangent leurs vêtements, hissant leurs dépouilles au-dessus d'eux. Ainsi suspendus les mannequins laissent place à leurs doubles, corps alignés au sol, qui vont s'animer au long de la pièce, jusqu'à se libérer d'eux par le mouvement, et dans un final qui ne les ignore pourtant, car tout se passe comme si, par-delà tout fétichisme, bien que reconduisant une présence fantomatique, les mannequins représentaient « la part d'ombre de chacun des sept danseurs, lesquels portent leur double mort sur le dos et doivent s'en occuper $»^{5}$. Ce qui ouvre la danse repose donc sur une dialectique du vif et du mort, de l'animé et de l'inanimé, dont la production d'énergie vise à la dépense, au sens de Bataille, et tire vers l'archaïque immémoriel : «Pour moi, ce qu'on appelle le spectacle vivant est connecté à une dimension supérieure qui relève du sacré. Une forme de sacré en marge du religieux ${ }^{6}$. C'est que Christian Rizzo vise à travailler le vide par peur du néant. 


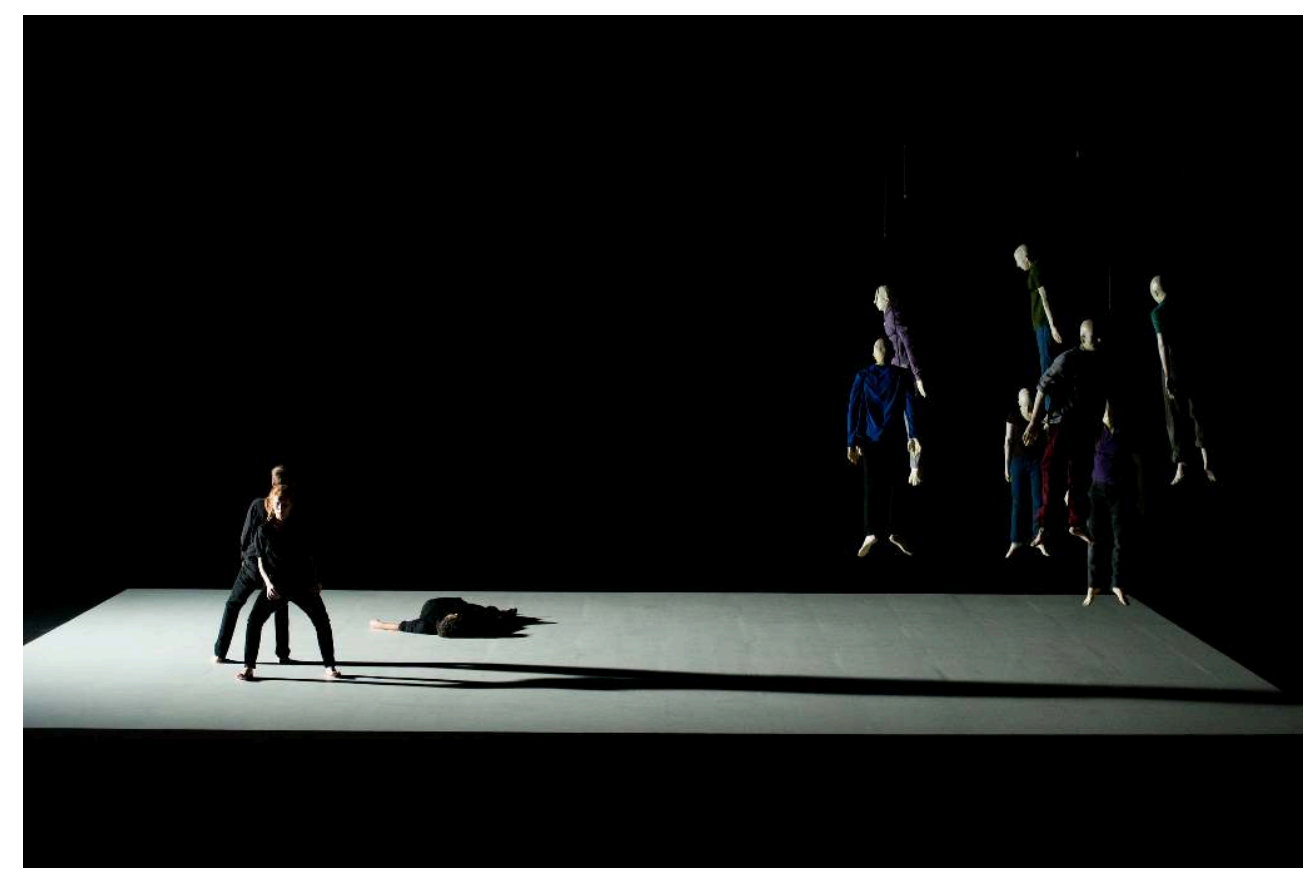

(c) Marc Dommage

4 À ces mannequins pendus, dépouilles mortelles de nos égos, qu'habillent et hissent les danseurs de leurs oripeaux pour se revêtir de cette autre peau d'ombre - danseurs au sol, mannequins oscillant dans la nuit - , Christian Rizzo n'oblige en rien à quelque danse macabre. Tout juste les voit-on ainsi que dans une résurrection du Jugement dernier de Lucas Signorelli ${ }^{7}$, soutirer de la pesanteur quelques nouvelles formes, quittant l'horizontalité du sol dans la quête d'une verticalité gagnée. Ainsi que les ombres portées des mannequins, les danseurs tout de noir vêtus, émergent du sol, rampant, roulant, avançant à quatre pattes, osant une chandelle, une arcature de dos, pour se redresser et prendre figure de marcheurs. L'éclairage de Caty Olive, la partenaire de toujours, fait alors merveille qui donne à lire l'entrelacement de leurs pas en ombres rasantes au sol. Lorsqu'une lumière zénithale jette les ombres ramassées au plus serré des pas, en d'erratiques déambulations, advient la musique de Robin Rimbaud, alias Scanner, qui porte les premiers balbutiements de langue, avant que se révèle le plateau nu sous un éclair, fiat lux marquant le moment où les danseurs réinvestissent la scène à reculons. Car il s'agit dans ce retournement généralisé d'explorer la forme du duo face au groupe, dans le passage à l'autre voir le groupe livrer place au duo et ce, dans une écriture qui travaille le vide entre les interprètes. Puis, un duo de danseuses se détache des sept interprètes, investit lâcher-prise et relève, chute et redressement, explore sous la pulsation lancinante de la musique la tension d'un poids-contrepoids, expérimente le bord-à-bord des corps dans un toucher inspiré de Paxton, forme une arche mouvante jusqu'à l'écroulement. Dès lors trouver la verticalité devient l'enjeu de l'intervention du groupe, les corps passent des uns aux autres jusqu'à dessiner une nouvelle confrontation sous le regard des danseurs alignés, un duo homme-femme. La voix de Monica Vitti dans l'Avventura d'Antonioni (1960) se glisse entre les pulsations rythmiques de la musique électronique, une nouvelle grappe humaine se constitue où se lisent d'impossibles échanges de couples jusqu'à 
épuisement. Seul persiste l'affrontement de deux danseurs, duo improbable, apposant sa résistance à la musique de Scanner.

Dans cette fabrique du duo resurgit la question tenace de Christian Rizzo : l'autre peutil être un double? Mais par le duo passe, aussi, l'incertitude du dubius, contre toutes les certitudes, au bénéfice d'un bene facere qui vise à condenser les gestes pour libérer leur charge émotionnelle. Loin de tout bénéfice d'inventaire, loin du spectaculaire: «en tant que chorégraphe, je n'ai rien à vendre, je n'ai pas de message politique à faire passer ni de croyance à inculquer aux autres : mon bénéfice, c'est le doute $»^{8}$.

Plus tard, placés au bord du plateau, les danseurs s'élanceront soudain, traverseront la scène en grappe humaine, en d'incessants échanges de duo croisant les appuis et les portés, balayant l'espace de tournoiements et de bras tendus. Les mannequins en surplomb descendus sur scène, portés par les danseurs seront alignés au sol en fond de scène, face au public, comme l'étaient les danseurs au début de la pièce. $7+7$ : sous la musique, en accéléré, l'oscillation des danseurs cette fois, ainsi qu'un mur humain vibrant au-dessus de leurs doubles inanimés, entame alors une chaîne rythmique, martelant l'espace de son archaïque pulsation. Mains jointes et bras tendus, laissant monter en eux une énergie tellurique, ils s'engagent autour des mannequins dans une danse ancestrale, en une bourrée occidentale mêlée de rythme africain, ainsi qu'on honorerait les morts en une ronde ensorcelée, jusqu'à briser le cercle vertueux par une diagonale vivante, et se retourner pour disparaître dans le nocturne profond, offrant sous les rampes de lumières, en un dernier éclair, exposées, les dépouilles des disparus. Toute existence humaine s'affirme sur un fond de disparition où la présence s'inscrit en creux d'un oubli qui se veut célébration. Ce qui nous tient en mouvement, encore.

\section{NOTES}

1. Christian Rizzo est, rappelons-le, un artiste polymorphe (danseur, chorégraphe, scénographe, styliste, et ancien rocker).

2. Rosita Boisseau, « Place au plaisir et à l'explosion physique », Le Monde, 9 juillet 2013.

3. Christian Rizzo, « Ce qui a déjà commencé... ", propos recueillis par Stéphane Malfettes, 26 octobre 2011 : http://www.lassociationfragile.com.

4. Idem.

5. Ibidem.

6. Ibidem.

7. Lucas Signorelli, La Résurrection de la chair, cathédrale d'Orvieto, chapelle San Brizio, 1499-1502.

8. Christian Rizzo, « Ce qui a déjà commencé... », art. cit. 